\title{
Acoustic Immittance, Absorbance, and Reflectance in the Human Ear Canal
}

\author{
John J. Rosowski, Ph.D. ${ }^{1}$ and Laura Ann Wilber, Ph.D. ${ }^{2}$
}

\begin{abstract}
Ear canal measurements of acoustic immittance (a term that groups impedance and its inverse, admittance) and the related quantities of acoustic reflectance and power absorbance have been used to assess auditory function and aid in the differential diagnosis of conductive hearing loss for over 50 years. The change in such quantities after stimulation of the acoustic reflex also has been used in diagnosis. In this article, we define these quantities, describe how they are commonly measured, and discuss appropriate calibration procedures and standards necessary for accurate immittance/reflectance measurements.
\end{abstract}

KEYWORDS: Admittance, impedance, immittance, tympanometry, stapes reflex

\begin{abstract}
Learning Outcomes: As a result of this activity, the participant will be able to (1) describe impedance and how it is related to acoustic reflectance and absorbance, and (2) describe the basic techniques for measuring immittance and reflectance and be able to list the standards associated with these measures.
\end{abstract}

Although physicians and audiologists have used bone conduction testing for over a century to help determine the amount of conductive hearing loss in a patient, it has only been in the past 50 years or so that attempts have been made to determine the nature of the conductive hearing loss without actually opening the ear. Initially two parallel tracks were taken-one to determine how much sound penetrated the eardrum (acoustic impedance) and the other to determine if the eardrum was moved by contraction of the middle ear muscles (acoustic reflex). Related techniques for independently assessing sound conduction through the eardrum and middle ear have since been developed, notably tympanometry, which allows relatively easy separation of the contribution of the air in the ear canal from the
${ }^{1}$ The Eliason Professor of Otology and Laryngology, Harvard Medical School, Massachusetts Eye and Ear Infirmary, Boston, Massachusetts; ${ }^{2}$ Department of Communication Sciences and Disorders, Frances Searle Building, Northwestern University, Evanston, Illinois.

Address for correspondence: Laura Ann Wilber, Ph.D., Communication Sciences and Disorders, Frances Searle Building, 2240 Campus Drive, Northwestern University, Evanston, IL 60208 (e-mail: 1-wilber@northwestern.edu).
Standardization and Calibration Part 2: Brief Stimuli, Immittance, Amplification, and Vestibular Assessment; Guest Editor, Robert Burkard, Ph.D.

Semin Hear 2015;36:11-28. Copyright (C) 2015 by Thieme Medical Publishers, Inc., 333 Seventh Avenue, New York, NY 10001, USA. Tel: +1(212) 584-4662.

DOI: http://dx.doi.org/10.1055/s-0034-1396924.

ISSN 0734-0451. 
measured impedance, and reflectance, which tells us how much sound is reflected from the eardrum. At present there is no national standard that yields absolute values for any of these procedures-aural acoustic impedance, aural acoustic admittance, tympanometry, acoustic reflex, or acoustic reflectance. However, there are American National Standards Institute (ANSI) and International Electrotechnical Commission (IEC) standards that describe procedures for calibrating the equipment used to make most of these measurements (with the exception of reflectance). The ANSI standard (ANSI S3.39-1987) was developed in 1987 and has been reaffirmed several times since then, but it has not been revised. The IEC standard (IEC 60645-5) was adopted in 2004.

In this article, we discuss some of the procedures for calibrating equipment used in measurements of acoustic immittance, reflectance, tympanometry, and acoustic reflex and some of the theoretical and mathematical bases of these measures. The discussion also will show some of the problems inherent in these measures as currently defined and used.

\section{BACKGROUND AND HISTORY}

To understand the problem, we should remember that when acoustic impedance and acoustic reflex measures were first introduced in the 1960s and early 1970s, although there were tuning fork tests, there was no standard for calibrating bone conduction testing (even though it was provided with most audiometers). Furthermore, computed tomography, magnetic resonance imaging, and other imaging procedures were not yet available for assessing middle ear problems. Thus, the surest ways to determine the presence and etiology of a conductive hearing loss was to otoscopically assess and surgically open the middle ear.

Work by Zwislocki designed to understand the physical characteristics of the ear ${ }^{1}$ led to the development of an acoustic impedance bridge, an acoustic impedance-measuring device used to assess how sound power entered the middle ear. Later, Zwislocki and Feldman ${ }^{2}$ showed that various conductive lesions yielded different acoustic impedance results. This acoustic bridge, which is no longer commercially available, allowed one to determine the resistance and reactance components of impedance at various frequencies. The bridge required the clinician to physically measure the volume of the ear canal, using warmed alcohol, and then to compare the sounds produced by a sound source coupled to the ear canal with the sound produced in a reference load of known acoustic impedance by the same source. The reference load was constructed from a series of tubes of controllable volume and cross section, which permitted the operator to dial up impedances with well-defined resistance and reactance. The sound source, the ear canal, and the reference impedances were so arranged that the sound produced in the two loads canceled one another when the reference impedance and the ear canal impedances were equal. The operator adjusted the reference impedance until cancellation occurred, then read the settings on the reference impedance and corrected for the impedance due to the volume of air in the ear canal to define the impedance of at the patient's eardrum.

Although the acoustic bridge was demonstrated to identify the aural impedances associated with various middle ear pathologies, ${ }^{2}$ practical problems associated with this method (e.g., measuring the volume of the ear canal and the development of reference impedances that matched the ear's impedance over a broad frequency range) led to the development of new electroacoustic instruments to determine the impedance at the eardrum. Those instruments typically measured the impedance at low frequencies, usually $226 \mathrm{~Hz}$ and, in fact, the ANSI standard only requires that modern instruments produce measurements for that frequency. Around the same time, that Zwislocki began his work, work in Europe by Terkildsen and Thomsen ${ }^{3}$ and Terkildsen and Nielsen ${ }^{4}$ showed that one could change the impedance at the eardrum by altering the static pressure in the ear canal.

At this point, it should be made clear that although the principles of acoustics easily describe the acoustic impedance of hard-walled cylindrical tubes or cavities, the ear canal is neither hard walled nor truly cylindrical. Thus, although impedance measurements made in the canal relate (and correlate well, at least at frequencies below $500 \mathrm{~Hz}$ ) to the impedance 
at the eardrum, they can be significantly influenced by the dimensions and shape of the ear canal and the stiffness and density of the ear canal wall. This limitation is a serious problem when trying to measure the impedance of the infant ear, because their canal walls are quite flaccid. ${ }^{5,6}$ This is less of a problem in older children and adults, ${ }^{7}$ and the pressure variationbased tympanometric procedure described by Terkildsen and his colleagues ${ }^{3,4}$ for separating the movement of the eardrum in the ear canal from the compressibility of the air in the residual canal depends on the constancy of the ear canal volume regardless of static pressure.

Finally, in this same period, Djupesland ${ }^{8,9}$ and Borg ${ }^{10}$ discovered that contractions of the stapedius muscle altered the impedance at the eardrum. This led to the observation that impedance measurements could provide insight into the mechanics of the middle ear, as well as the neural pathways that lead to the contraction of the stapedius muscle. In the latter circumstance, the detailed characteristics of the ear canal were not so important because one need only detect a change in impedance due to a reflex muscle contraction and detect whether or not the contraction diminished over time. ${ }^{11}$ Regarding the differential diagnosis of conductive hearing loss, the presence of sound-induced changes in impedance produced by the reflex is an indication of ossicular normality, and the lack of such an impedance change is an indication of ossicular abnormality and conductive hearing loss.

The complexities of the ear canal and the shape of the eardrum make it difficult to ascertain precisely the impedance of the eardrum and the attached middle and inner ear. As an aside, when work first began on developing standards for acoustic impedance, the geometric assumptions needed to compute the impedance, within the complex-shaped ear canal and the nonplanar shape of the eardrum, led the standard committee to question whether one could actually measure acoustic impedance at the eardrum in humans. This discussion resulted in the coinage of the term aural acoustic immittance, which is a combination of impedance and admittance and specifically relates to the ear. But, just as we know that measuring sound in a coupler for pure tone audiometry does not tell us the exact level of the sound reaching the eardrum, let alone what the stimulus is within the inner ear or reaching the auditory cortex, such measurements do ensure (1) our instrument has not changed over time, (2) our instrument measures the same thing as a similar instrument, (3) individual results do or do not that change over time, and (4) accurate comparisons to national and international norms. Although it is often presumed that acoustic reflectance is not as easily contaminated by the vagaries of ear canal and eardrum structure, there is no standard at this time for measuring reflectance, such that calibration methods are determined by the manufacturer and comparisons between different devices rely on interested and involved clinicians and scientists (e.g., Shahnaz et $\mathrm{al}^{12}$ ).

\section{STANDARDS FOR ACOUSTIC IMMITTANCE AND REFLECTANCE MEASUREMENT}

As we have seen, the measurement of acoustic immittance and reflectance in the human ear canal are common tools in the investigation and differential diagnosis of the causes of hearing loss, and there are several U.S. and international standards relevant to this use, including:

ANSI S1.1-2013 Acoustic Terminology. As its title suggests this standard is relevant to both the definitions of the different quantities discussed, and to the nomenclature used in this article.

ANSI S3.39-1987 [R 2012] Specifications of Instruments to Measure Aural Acoustic Impedance and Admittance (Aural Acoustic Immittance). (Note: The use of the extension [R 2012] means that this was reaffirmed in 2012.) This standard references the precursors to ANSI S1.1-2013 and defines a relevant subset of the important quantities and nomenclature. It also describes specifications and calibration apparatus relevant to traditional low-stimulusfrequency tympanometers and devices for evaluating the contraction of the middle ear muscles.

IEC 60645-5 Electroacoustics-Audiometric Equipment-Part 5: Instruments for the Measurement of Aural Acoustic 
Impedance/Admittance, adapted by the International Electrotechnical Commission in November of 2004. This standard has substantial overlap with ANSI S3.39-1987.

These standards are listed in Table 1 and are referred to throughout this article. Terminology defined in the standards will first be introduced by italicized text, followed by a reference to the appropriate standard. Table 2 summarizes the symbols that refer to the physical quantities associated with determining acoustic impedance and admittance.

\section{THE QUANTIFICATION OF SOUND: SOUND PRESSURE AND VOLUME VELOCITY}

Sound in a medium such as air is associated with net repeated forward and backward variations in the position of small collections of molecules of the medium (Fig. 1). Each particle contains a quantity of molecules large enough that random molecular motions average to zero, thereby revealing the net average motion imposed on the particles by sound. As the driven particles move back and forth in the sound field, toward and away from each other, the density of the particles in the field varies, and associated with the variations in density is a variation in local pressure. The root mean square (rms; a measure of the time averaged absolute value of a quantity) pressure variation around the baseline pressure (the static pressure, 2.58: ANSI S1.1-2013) defines the magnitude of the sound pressure ( $P$, 2.59: ANSI-S1.1-2013), which is easily measured by a microphone. The standard unit of sound pressure is the pascal $(\mathrm{Pa})$. The rms sound pressure is the root of the mean of the square of the time-dependent deviations around the static baseline pressure and is mathematically equivalent to the standard deviation around the baseline. The magnitude of motion of the particles of air is more difficult to measure, but is usually defined in terms of the rms particle velocity (2.63: ANSI S1.1-2013) with units of meters per second $\left(\mathrm{m} / \mathrm{s}^{-1}\right)$. When dealing with enclosed spaces, such as tubes, we can quantify the particle motion in terms of a volume velocity ( $U, 2.65$ : ANSI S1.1-2013), when we assume, usually with a good degree of accuracy, the velocity of the air particles is constant across a cross section of the tube that is orthogonal to the direction of sound propagation (a plane wave, 5.14:ANSI S1.1-2013), the volume velocity equals the product of the rms velocity of the air particles and the crosssectional area of the tube and has units of (cubic meters per second, $\mathrm{m}^{3}-\mathrm{s}^{-1}$; Fig. 1).

\section{ACOUSTIC IMMITTANCE: IMPEDANCE OR ADMITTANCE}

The acoustic impedance and admittance are related quantities that depend on the ratio of sound drive (the sound pressure) and sound flow (the volume velocity of sound). When defined at the eardrum, the entrance to the middle ear, these quantities help determine the work done by the sound in moving the eardrum and ossicles. In the case of tonal stimulation at varied stimulus frequency $(f)$ in a tube or ear canal, the ratio of the sound pressure $P(f)$ to the volume velocity in the tube (or canal) $U(f)$, defines the acoustic impedance $Z_{a}(f)$ (6.41: ANSI S1.1-2013) at the point of measurement, which generally depends on $f: Z_{a}(f)=P(f) / U$ $(f)$. The acoustic impedance describes the sound

Table 1 Standards That Are Relevant to Measures of Acoustic Impedance/Admittance/ Reflectance/Absorbance

ANSI S1.1-2013

ANSI S3.39-1987 (R 2012)

IEC 60645-5 Ed. 1: 2004
American Nation Standard Acoustic Terminology
American National Standard Specifications of Instruments to
Measure Aural Acoustic Impedance and Admittance (Aural Acoustic
Immittance)
Electroacoustics-Audiometric equipment_-Part 5: Instruments for
the measurement of aural acoustic impedance/admittance

ANSI, American National Standards Institute; IEC, International Electrotechnical Commission. 
Table 2 List of Terms and Abbreviations

\begin{tabular}{|c|c|c|c|}
\hline Quantity & Symbol & SI Units & Derived Units \\
\hline Acoustic admittance & $Y_{a}$ & $m^{3}-\mathrm{Pa}^{-1}-\mathrm{s}^{-1}$ & Acoustic siemens \\
\hline Acoustic conductance & $g_{a}$ & $\mathrm{~m}^{3}-\mathrm{Pa}^{-1}-\mathrm{s}^{-1}$ & Acoustic siemens \\
\hline Acoustic susceptance & $b_{a}$ & $\mathrm{~m}^{3}-\mathrm{Pa}^{-1}-\mathrm{s}^{-1}$ & Acoustic siemens \\
\hline Admittance phase angle & $\varphi_{a}$ & Radians & \\
\hline Acoustic impedance & $Z_{a}$ & $\mathrm{~Pa}-\mathrm{m}^{-3}-\mathrm{s}^{-1}$ & Acoustic ohms \\
\hline Acoustic resistance & $r_{a}$ & $\mathrm{~Pa}-\mathrm{m}^{-3}-\mathrm{s}^{-1}$ & Acoustic ohms \\
\hline Acoustic reactance & $x_{a}$ & $\mathrm{~Pa}-\mathrm{m}^{-3}-\mathrm{s}^{-1}$ & Acoustic ohms \\
\hline Impedance phase angle & $\varphi_{z}$ & Radians & Radians \\
\hline Acoustic mass (inertance) & $M_{a}$ & $\mathrm{~Pa}-\mathrm{m}^{-4}$ & \\
\hline Acoustic compliance & $C_{a}$ & $\mathrm{~m}^{3}-\mathrm{Pa}^{-1}$ & \\
\hline Equivalent volume & $V_{e}$ & $\mathrm{~m}^{3}$ & $1 \mathrm{~m}^{3}=10^{6} \mathrm{~cm}^{3}$ \\
\hline Ambient pressure & $p_{s}$ & $N-m^{-2}$ & $\mathrm{~Pa}$ \\
\hline Sound pressure & $P$ & $\mathrm{~N}-\mathrm{m}^{-2}$ & $\mathrm{~Pa}$ \\
\hline Sound pressure phase angle & $\varphi_{p}$ & Radians & Radians \\
\hline Particle velocity & & $m-s^{-1}$ & \\
\hline Volume velocity & $U$ & $m^{3}-s^{-1}$ & \\
\hline Sound pressure phase angle & $\varphi_{u}$ & Radians & Radians \\
\hline Static density & $\rho_{0}$ & $\mathrm{~kg}-\mathrm{m}^{3}$ & \\
\hline Velocity of propagation & $c$ & $m-s^{-1}$ & \\
\hline Sound pressure reflection coefficient & $R$ & Dimensionless & Dimensionless \\
\hline Phase of the sound pressure reflection coefficient & $\varphi_{R}$ & Degrees & Degrees \\
\hline Power reflection coefficient & $R$ & Dimensionless & Dimensionless \\
\hline Sound absorbance & $A$ & Dimensionless & Dimensionless \\
\hline ratio of specific heat of a gas & $\gamma$ & Dimensionless & Dimensionless \\
\hline
\end{tabular}

Abbreviation: SI, System International.

pressure needed to produce a unit measure of volume velocity in the tube, and the impedance value is related to the physical properties of the fluid and the container that restricts fluid motion. This is analogous to the generalization of Ohm's law to time varying electric signals, where the time-varying voltage ( $E$, the analog of sound pressure) is related to the current ( $I$, the analog of the volume velocity) by the electrical impedance $\left(Z_{e}(f)\right)$, where the electrical impedance can be defined as the ratio of measurements of the voltage and the current: $Z_{e}(f)=E(f) / I(f)$.

In a short air-filled tube (the length of the tube is less than 0.1 of a wavelength) open at both ends, the mass of the air particles dominates the acoustic impedance. The compressibility or compliance of the air dominates the impedance in a short tube that is closed at the far end (e.g., by a higher impedance eardrum). In narrow air-filled tubes, the viscosity of the air can contribute to the impedance. In longer tubes, the impedance depends on a combination of mass, compressibility, and viscous and other losses. The unit of acoustic impedance is the acoustic ohm, which describes the ratio of sound pressure (in $\mathrm{Pa}$ ) per volume velocity $\left(\mathrm{m}^{3}-\mathrm{s}^{-1}\right)$, where 1 acoustic ohm $=1 \mathrm{~Pa}^{-\mathrm{m}^{-3}}$-s. Acoustic admittance $Y_{a}(f)$ is the ratio of volume velocity to sound pressure, that is, $Y_{a}(f)=1 / Z_{a}(f)$, and describes the volume velocity required to produce a unit of sound pressure (6.47: ANSI S1.12013). The units of acoustic admittance are acoustic siemens, where 1 acoustic siemen $=1$ $\mathrm{m}^{3}-\mathrm{s}^{-1}-\mathrm{Pa}^{-1}$. Admittance and impedance are often grouped under the rubric immittance (6.18: ANSI S1.1-2013).

Although we have defined the impedance and admittance in terms of the frequencydependent $P(f)$ and $U(f)$ associated with tonal stimulation, we have simplified this description by ignoring the phase of the measured pressures 


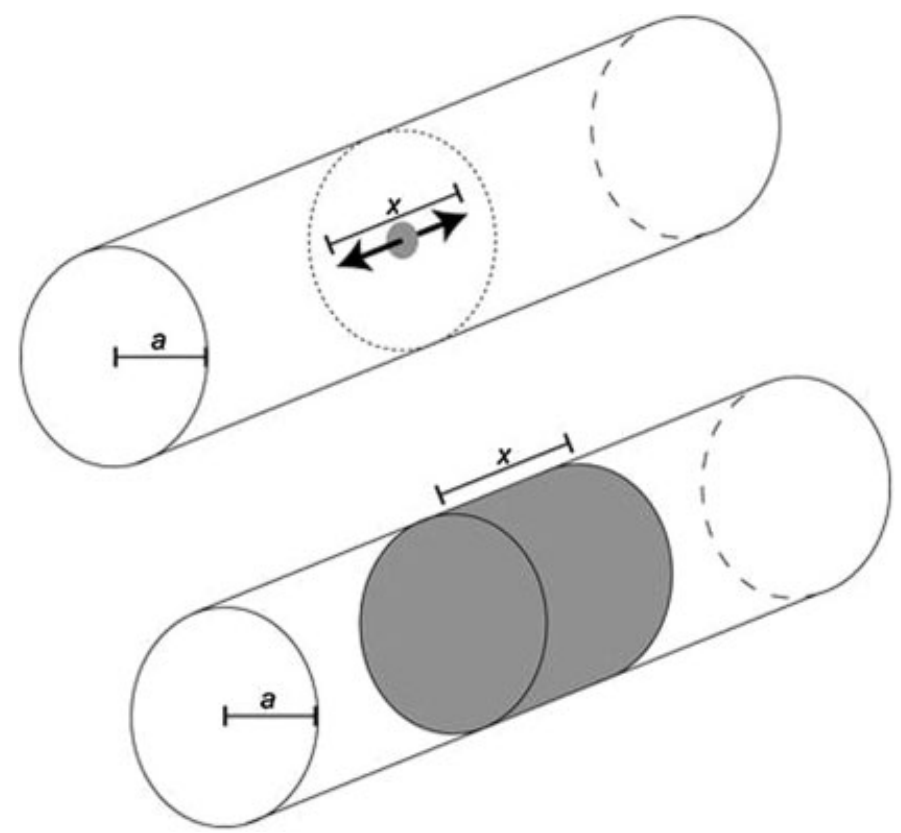

Figure 1 Particle velocity and volume velocity. (Top) A 100-Hz tone propagating down a uniform cylindrical air-filled tube of radius a, sets a "particle" of the air medium into back and forth motion, with a peak-to-peak (3.15: ANSI S1.1-1994) particle displacement equal to $x$ meters. The root mean square (rms) displacement of the particle equals $x /(2) \sqrt{2} \sqrt{2}=x / 2.82 \mathrm{~m}$. The rms back-and-forth velocity of the particle is the product of the rms displacement and the radian frequency of the tone: rms velocity ( $V$, with units of $\mathrm{m} / \mathrm{s})=2 \pi 100(\mathrm{~Hz})$ $x / 2.82(\mathrm{~m})$. (Bottom) Calculation of the volume velocity assumes that all of the air particles in the same crosssectional plane of the tube (the dotted line in the top tube) move together (the uniform-plane wave approximation). The gray region in the bottom tube describes the volume displaced by the plane of particles as it moves back and forth in the sound wave and has a volume equal to the product of the peak-to-peak displacement $x$ and the area of the tubal cross section $\pi a^{2}$. The rms volume displaced by the particles as they move back and forth is the product of the rms particle displacement and the area of the tube $=(x / 2.82)$ $\pi a^{2}$. The rms volume velocity (the time rate of change of the displaced volume) equals the product of the rms velocity and the area of the tube $=(2 \pi 100 \times / 2.82) \pi a^{2}$, with units of $\mathrm{m}^{3} / \mathrm{s}$.

and volume velocities. In a linear system, a sinusoidal input produces sinusoidal responses, and the responses produced by a tonal stimulus can differ from the input in terms of the magnitude (which we quantify in terms of the rms amplitude), and phase (which describes the relative timing of the sinusoidal responses, 2.23: ANSI S1.1-2013). A complete description of the system's response requires knowledge of both of these response properties. In particular the impedance magnitude $\left|Z_{a}(f)\right|$ is defined by the ratio of the magnitudes of the sound pressure $|P(f)|$ and the volume velocity $|U(f)|$. The impedance phase angle $\varphi_{Z}(f)$ equals the difference between the response phase of the sound pressure $\varphi_{P}(f)$ and the phase of the volume velocity $\varphi_{U}(f)$.
An alternative form that quantifies the magnitude and phase angle of impedance and admittance is to describe these quantities in terms of complex numbers with a real part and an imaginary part, for example, $Z_{a}(f)=r_{a}(f)+i$ $x_{a}(f)$, where $i$ is the imaginary number equal to

$$
\begin{aligned}
\sqrt{-1} z_{a}(f) & =\sqrt{r_{a}^{2}(f)-x_{s}^{2}(f)}, \text { and } \\
\phi_{s} & =\tan ^{-1}\left(x_{u}(f) / r_{a t}(f)\right) .
\end{aligned}
$$

This relationship is graphically described in Fig. 2. The real part of the complex impedance is the resistance $r_{a}$ and the imaginary part is the reactance $x_{a}$, where both of these factors can vary with stimulus frequency. Similarly, we can define the admittance in terms of its conductance $g_{a}$ and susceptance $b_{a}$, where $Y_{a}(f)=g_{a}(f)+i b_{a}(f)$. It is important to realize that $Y_{a}(f)=1 / Z_{a}(f), r_{a}(f)$ 


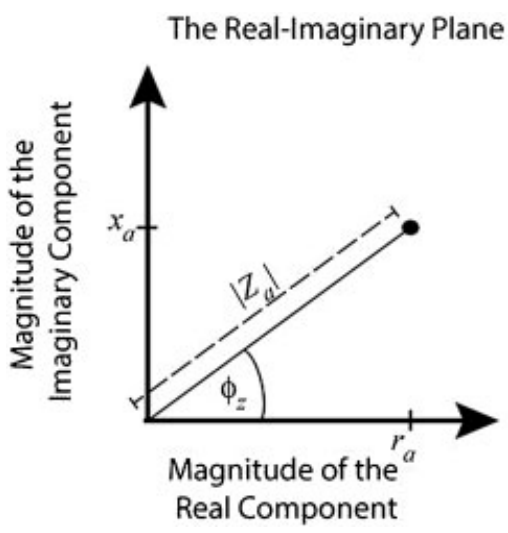

Figure 2 An illustration of the relationship between the real and imaginary components of a complex number and the magnitude and angle of the complex value. The real $r_{a}$ and imaginary $x_{a}$ components of a complex impedance $Z_{a}$ are illustrated as the two coordinate values on the real-imaginary plane. The complex value $\left(Z_{a}=r_{a}+i x_{a}\right)$ is represented by the plotted point. The length of the line connecting the point to the origin is the magnitude of the complex value $\left|Z_{a}\right|$. The angle between the line and the "real" axis is the phase angle of the complex value $\varphi Z_{a}$.

does not generally equal $1 / g_{a}(f)$ nor does $x_{a}(f)$ generally equal $1 / b_{a}(f)$.

There is another common unit of admittance magnitude that is applied to admittances governed either by the compressibility of the medium or the compliance of the tubal boundaries. The admittance of the human eardrum or tympanic membrane (TM) at frequencies below $400 \mathrm{~Hz}$ is dominated by the compliance of the TM, its coupled ossicles, and their supporting ligaments. Such compliant admittances can be quantified in terms of the equivalent volume $V_{e}$ of air (5.11: ANSI S3.39-1987) that has an identical admittance magnitude. Specifically, the magnitude of admittance of a volume of air within a small closed cavity (where small is determined by comparisons of the cavity dimensions to the wavelength of the sound, and is generally defined as linear dimensions that are less than one-tenth of a wavelength) can be determined from the following:

$$
\left|Y_{a}\right|=2 \pi f \frac{\text { Volume }}{\gamma p_{S}} \text {, (Eq. 1) }
$$

where $f$ is frequency in $\mathrm{Hz}$; Volume is defined in $\mathrm{m}^{3}, \gamma$ is the ratio of specific heat $\sim 1.4$ for the diatomic ideal gases in air, and $p_{s}$ is the static ambient atmospheric pressure of $\sim 100,000 \mathrm{~Pa}$. If instead of System International (SI) units, we use the older centimeter-gram-second units (where $\left|Y_{a}\right|$ is defined in terms of $\mathrm{cm}^{5}-\mathrm{s}^{-1}$ dyne ${ }^{-1}$, Volume is defined in $\mathrm{cm}^{3}$, and $p_{\mathrm{S}}=1$ million dyne- $\mathrm{cm}^{-2}$ ) and evaluate the expression at $226 \mathrm{~Hz}$, we find that an air volume of $1 \mathrm{~cm}^{3}$ gives rise to an admittance of 1 milli-cgs admittance unit, the mho, where $1 \mathrm{cgs}$ mho $=1 \mathrm{~cm}^{5}-\mathrm{s}^{-1}$-dyne ${ }^{-1}$. Simply put, at $226 \mathrm{~Hz}$, an admittance magnitude of 1 millimho $(=1$ $\mathrm{mho}^{-3}$ ) is produced by an air-filled cavity that is $1 \mathrm{~cm}^{3}$ in volume, an admittance magnitude of $0.5 \mathrm{mho}^{-3}$ is produced by an air-filled cavity that is $0.5 \mathrm{~cm}^{3}$ in volume, and so on. Because the admittance produced by a closed air space depends on $f$, the equality of the value of the equivalent volume in $\mathrm{cm}^{3}$ and the value of the admittance in $\mathrm{mho}^{-3}$ only occurs at $226 \mathrm{~Hz}$. Indeed the simplifying equality at that frequency is the root of the ANSI and IEC standards' call for measuring tympanograms at $226 \mathrm{~Hz}$.

\section{PRESSURE REFLECTANCE}

Pressure reflectance measured in the ear canal is another measure of the difficulty in setting the eardrum and ossicles into motion, where large amounts of sound pressure reflected from the eardrum are associated with reduced eardrum motions. Mathematically, acoustic reflectance is related to the acoustic impedance, in that reflections occur at boundaries where the acoustic impedance changes. For example, consider a cylindrical air-filled tube of radius $a$ (such as in Fig. 1) terminated at one end by an acoustic impedance of value $Z_{a, T}(f)$ with magnitude and angle. The subscript $T$ is used to define the location at the termination of the tube. Sound waves that propagate from the open end of the tube toward the termination are carried by the air channel, where sound propagation down the channel is related to the tube's characteristic acoustic impedance $Z_{0}$, which equals the product of the static density of air $\left(\rho_{0}\right)$ and the propagation velocity of sound $(c)$ divided by the cross-sectional area of the tube, that is, $Z_{0}=\left(\rho_{0} c\right) /\left(\pi a^{2}\right)$. The sound pressure reflection coefficient (10.05: ANSI S1.1-2013) at the termination $R_{T}(f)$ is a complex quantity 
(with a magnitude and phase) related to $Z_{0}$ and $Z_{a, T}(f)$ by the following:

$$
R_{T}(f)=\frac{Z_{a, T}(f)-Z_{0}}{Z_{a, T}(f)+Z_{0}} \text {. (Eq. 2) }
$$

$R_{T}(f)$ defines the ratio of the sound pressure in the wave reflected from the termination to the sound pressure in the wave moving toward the termination. ${ }^{13,14}$

The sound pressure reflection coefficient also can be quantified any distance $x$ from the termination $R(x, f)$ that is still within the tube. In the case of a straight tube of uniform crosssectional area throughout (e.g., Fig. 1), the magnitude of the pressure reflectance does not vary with $x,|R(x, f)|=\left|R_{T}(f)\right|$, but the angle of the pressure reflectance varies regularly with

$x, \phi_{R}(x, f)=\phi_{R}(x, f)+\left(\left(\frac{4 \pi}{c}\right)\right) x .^{15}$ Therefore,

knowledge of the dimensions of the tube and the magnitude and phase of the terminating impedance $Z_{a, T}(f)$ can be used to compute the pressure reflectance at any $x$ position in the tube. Conversely, knowledge of the tube dimensions, the distance $x$ between the measurement point and the termination, and the magnitude and angle of $R(x, f)$ are needed to compute the terminating impedance $Z_{a, T}(f)$.

\section{POWER REFLECTANCE AND ABSORBANCE}

In the previous section, we noted that in a straight tube of uniform cross section, the magnitude of the pressure reflection coefficient is invariant with distance from the reflecting surface, but the angle of the coefficient varies regularly with that distance. There is another coefficient, the sound power reflection coefficient (10.04: ANSI S1.1-2013), that has no angle and remains constant throughout the tube, with a value equal to the square of the magnitude of the pressure reflection coefficient at the termination: $\mathcal{R}(f, x)=\left|R_{T}(f)\right|^{2}$. The constancy of the power reflection coefficient in a uniform tube is an attractive feature that allows assessment of the power reflectance at the tube's termination from any location within the tube. However, deviations from a uniform tube can influence the power reflection coefficient ${ }^{16}$ and complicate the relationship between the power reflectance at the point of measurement and at the termination. ${ }^{15}$

The opposite of power reflectance is power absorption, where the sound power absorption coefficient (10.02: ANSI S1.1-2013) describes the fraction of the sound power incident on a surface that is absorbed, rather than reflected, where $\mathcal{A}(f)=1-\mathcal{R}(f)$. Like the power reflection coefficient, in a straight uniform tube $A(f)$ is independent of the position $x$ in the tube and dependent solely on the absorption coefficient at the terminating reflecting surface.

\section{IMPEDANCE/ADMITTANCE AND REFLECTANCE/ABSORBANCE ARE DIFFERENTLY AFFECTED BY THE EAR CANAL}

In most clinical instruments, measurements of the aural impedance and reflectance (and their associated quantities admittance and absorbance) are made via an insert earphone and microphone placed in the ear canal at a distance of 1 to $2 \mathrm{~cm}$ from the eardrum. The residual ear canal air space between the eardrum and the measurement sight affects the measured impedance or reflectance in different manners. The added air space acts to absorb and store part of the sound energy introduced by the earphone, such that only a fraction of the stimulus sound energy works against the lateral surface of the eardrum to drive the middle ear and cochlea.

In terms of impedance, the added load of the ear canal air acts like an impedance in front of the impedance measured at the eardrum. In most cases, this ear canal contribution to the measured impedance is significant, and the combined impedance needs to be compensated for the presence of the ear canal before one can effectively describe the aural impedance at the eardrum. The need for this compensation was the reason Zwislocki's acoustic bridge measurements in patients were accompanied by measurements of the volume of the patients' ear canal; knowledge of the ear canal volume allows some simple approximations of its contribution to the measured impedance, however, such simple approximations are only accurate at frequencies less than $1 \mathrm{kHz} .{ }^{17}$ 
The effects of the ear canal on the reflectance measured in the ear canal are more subtle. Theory tells us the magnitude of the pressure reflectance $|R(x, f)|$ together with the power reflectance $R(f)$ and absorbance $A(f)$ measured anywhere within a tube of uniform or slowly varying cross section equals the magnitude of the pressure reflectance at the termination. ${ }^{15}$ Therefore, if the ear canal fit those geometrical constraints (uniform or slowly varying cross section), then the power reflectance measured at any point in the ear canal, would equal the power reflectance at the eardrum. However, real ear canals only approximate these geometric constraints, and there is evidence that power reflectance and absorbance do vary when measured at different positions in an ear canal. ${ }^{16,18}$

\section{TYMPANOMETRY USES VARIATIONS IN STATIC PRESSURE TO HELP CORRECT FOR THE EAR CANAL}

All of the quantities we have introduced depend on the frequency of tonal stimulation, and in general measurement of these quantities at multiple frequencies increases the information available about the mechanoacoustic properties that constrain the behavior of the TM and middle ear. Early measurements of the immittance made in human ear canals were made over a range of frequencies using a series of pure tone stimuli that varied from 0.1 to $2 \mathrm{kHz},{ }^{2}$ and such measurements helped distinguish various forms of conductive hearing loss. The $2-\mathrm{kHz}$ limit in these studies was related to the accuracy of the immittance calibration and the methods used to compensate for the presence of the ear canal. It was these complications that led later studies ${ }^{19}$ to concentrate on one or two tonal stimuli of relatively low frequency and use variations in ear canal static pressure to help estimate the contribution of the ear canal.

These early tympanograms (5.22: ANSI S3.39-1987) plotted variations in sound pressure within the ear canal produced by a pure tone stimulus as a function of controlled variations in ear canal static pressure, where the pressure variations were on the order of \pm 300 $\mathrm{daPa}$ (equal to $\pm 3000 \mathrm{~Pa}$ and approximately equivalent to static pressures of $\pm 300 \mathrm{~mm}$ $\mathrm{H}_{2} \mathrm{O}$ ). Later devices were able to convert static pressure-induced variations in ear canal sound pressure into variations in immittance and its components of resistance, reactance, conductance, or susceptance ${ }^{20,21}$; however, these early tympanometers generally were restricted to measuring impedance at one or two low frequencies, usually 226 and $660 \mathrm{~Hz}$.

The common idea in any tympanogram is that the static pressure variation alters the immittance of the TM and middle ear without altering the contribution of the ear canal. More precisely, the common assumption is that the presence of significant static pressure in the ear canal rigidifies the TM and middle ear, such that the immittance measured with large static pressures is that of the ear canal alone. This high static pressure-determined ear canal contribution can then be subtracted from the combined ear canal and TM immittance measured with zero static pressure, with the immittance at the TM left as the remainder. One complication in this scheme is that the TM is not made completely rigid by static pressures. ${ }^{17,22}$ Another is that the method of simply subtracting the ear canal component of the immittance to reveal the immittance at the TM works best at low frequencies, where the ear canal immittance can be described by a single compliance; this simple calculation becomes inaccurate at higher frequencies where the distance between the measurement point in the ear canal and the TM becomes larger than 0.1 wavelengths. ${ }^{17}$ Finally, although the assumption of the invariance of the ear canal with varied static pressure appears accurate in adult humans, it has been shown to be inaccurate in infants, where the soft cartilaginous canal walls expand and contract with static pressure. ${ }^{23}$ These complications in correcting measurements of immittance for the presence of the ear canal are some of the arguments for the use of reflectance/absorbance, where these quantities appear less affected by the presence of the ear canal. Nonetheless, some reports suggest a utility for measurements of reflectance at varied ear canal sound pressure. ${ }^{24,25}$ 


\section{WIDEBAND VERSUS NARROWBAND MEASUREMENTS}

The information gathered from ear canal immittance measurements about the terminating immittance at the TM increases as measurements are made over a wider frequency range. Low-frequency estimates can help describe the stiffness of the middle ear, but measurements at higher frequencies appear more sensitive to the presence of fluid or other variations in the mass and resistance at the TM. ${ }^{26,27}$ An increased utility of measurements at higher frequencies has been demonstrated in infants, where the immittance of the ear canal walls are dominated by a large compliance at low frequencies. ${ }^{23,27}$ Immittance and reflectance measurements at frequencies between 0.5 and $4 \mathrm{kHz}$ appear most useful in differentiating ossicular disorders. ${ }^{28}$ Although the best estimates of immittance at the TM from ear canal measurements appear limited to frequencies between 0.2 and $4 \mathrm{kH}$, primarily due to complications in accounting for the presence of the ear canal, ${ }^{17}$ power reflectance and absorbance (which are less affected by the intervening ear canal) are routinely measured at frequencies as high as 6 to $8 \mathrm{kHz} \cdot{ }^{14,29}$

\section{ACOUSTIC REFLEX}

Immittance/reflectance tests of the acoustic reflex depend on measurements of one of these quantities made before and after presenting an ipsilateral or contralateral sound stimulus designed to evoke a contraction of the middle ear muscles. ${ }^{11}$ The basic clinical test uses a continuous single-tone probe stimulus (called the probe signal [5.15: ANSI S3.39-1987], usually of a frequency of $226 \mathrm{~Hz}$ and less than or equal to 90-dB SPL [7.3.5: ANSI S3.39-1987]) to measure the impedance/admittance. Then a stimulus tone or noise stimuli of moderate level is presented to either the ipsilateral or contralateral ear. The frequencies of the eliciting tones are usually the common audiometric frequencies (7.5.2: ANSI S3.39-1987). The noise may be either broadband or band-limited (7.5.3: ANSI S3.39-1987). The level of the eliciting tone-burst is adjusted to identify the sound level that just produces a noticeable change in the measured admittance. This is the threshold of the acoustic reflex (5.23.1: ANSI S3.39-1987). Once the threshold is identified, suprathreshold stimuli of longer duration can be used to quantify the time course of the reflex. ${ }^{11}$ Presence of the acoustic reflex is a positive indicator for an intact ossicular chain. Absence of the reflex is consistent with a conductive hearing loss (such as otosclerosis or interruption of the connection between the incus and stapes) or a neurologic disorder along the reflex pathway (such as a brainstem infarct or Bell's palsy of the facial nerve segment between the stapedius muscle and the brain stem). Wideband immittance and reflectance also have been used to estimate the threshold of the acoustic reflex. ${ }^{30-32}$ The thresholds estimated by these wideband techniques are $\sim 15 \mathrm{~dB}$ lower than the thresholds measured with a single $226-\mathrm{Hz}$ probe tone. The increased sensitivity of the wideband tests has been attributed to either better signal-to-noise ratio in such tests or to the frequency dependence of the effect of stapedial muscle contraction on middle ear immittance ${ }^{33,34}$ published in Fig. 5 of (Peake and Rosowski $\left.1997^{35}\right)$. The higher sensitivity of reflectance measurements to stapedial contraction may be significant when considering the possibility of iatrogenic hearing loss induced by the high stimulus levels ( $>100-\mathrm{dB}$ SPL) sometimes used to evoke the reflex in patients with hearing loss. ${ }^{30,36,37}$

\section{CALIBRATION METHODS}

\section{National and International Standards}

The worldwide acceptance of tympanometry as a measure of middle ear performance has led to the definition of national and international standards for the design and implementation of tympanometers, that is, ANSI S3.39-1987 and IEC 60645-5. Much of the verbiage in these standards revolves around the mechanism and specifications for changing the ear canal static pressure, both in terms of the range of the static pressure variations and the rate of change of these pressures. The standards are fairly open concerning the sound stimuli used to measure immittance, either tonal or broadband, with constraints placed on the sound levels used (the probe tone used to measure immittance should 
have a level of 90-dB SPL or less, 7.3.5: ANSI S3.39-1987). ${ }^{*}$ The methods are also fairly open concerning the technique for calibration, though they suggest the use of acoustic standards, made of rigid-walled cavities of some range of dimensions and equivalent volume, as reference immittances. Although useful, the standards are not very descriptive regarding calibration methods, and the suggestion of the dimensions of standard acoustic admittances are not practical when considering measuring immittance and reflectance at frequencies above a few $\mathrm{kHz}$. Therefore, in cases of measurements of wideband immittance measurements, the different manufacturers provide the hardware and describe procedures necessary to compute immittance and reflectance values from measurements of sound pressure in ear canals and other acoustic loads.

\section{Calibration of Equipment Ancillary to Immittance/Reflectance Measurements}

The areas where calibration of the tympanometers and acoustic reflex measuring devices should be checked are: (1) the frequency of the probe tone; (2) the impedance of the probe as measured in a cylindrical air-filled cavity $\left(1 \mathrm{~cm}^{3}\right.$ in size); (3) the air pressure (measured with a manometer or U-tube); (4) the characteristics of the tones used to measure the acoustic reflex-frequency, distortion, and level. The standard specifies what characteristics should be provided with different types of instruments. The different types are defined by the quantities that they measure and the functions they perform. Devices with the greatest range of functionality are type I devices (ANSI S3.39-1987).

The standard for aural acoustic immittance (impedance/otoadmittance) devices is ANSI S3.39-1987 (ANSI 1987). There is also an IEC Standard, IEC 61027 for measurement of aural acoustic impedance/admittance (IEC 1991). The ANSI standard describes four types of devices for measuring acoustic immittance (listed simply as types 1,2, 3, and 4; ANSI S3.39-1987). The specific minimum mandatory

\footnotetext{
Some more recent work suggests 90-dB SPL may be too intense, in that it may evoke the acoustic reflex or even cause temporary threshold shift. ${ }^{32}$
}

requirements are given for types 1,2 , and 3 . There are no minimum requirements for the type 4 device. Types 1, 2, and 3 must have at least a $226-\mathrm{Hz}$ probe signal, a pneumatic system (manual or automatic), a way of measuring static acoustic immittance, tympanometry, and the acoustic reflex. Thus, to check the acoustic immittance device one may begin by using a frequency counter to determine the frequency of the probe signal(s). The frequency should be accurate within $3 \%$ of the nominal value. The total harmonic distortion shall not exceed $5 \%$ of the fundamental when measured in an HA-1-type coupler (this is commonly called a $2-\mathrm{cm}^{3}$ coupler). The probe signal shall not exceed $90 \mathrm{~dB}$ as measured in that coupler. The range of acoustic-admittance and acoustic-impedance values that should be measurable varies with instrument type. The accuracy of the acoustic-immittance measurements should be within $5 \%$ of the indicated value, or $\pm 10^{-9}$ $\mathrm{cm}^{-3} / \mathrm{Pa}$ (0.1 acoustic mmhos), whichever is greater. The accuracy of the acoustic immittance measurement can be determined by connecting the probe to the test cavities and checking the accuracy of the output at specified temperatures and ambient barometric pressures. A procedure for checking the temporal characteristics of the acoustic-immittance instrument is described by Popelka and Dubno ${ }^{38}$ and by Lilly. ${ }^{39}$

Air pressure may be measured by connecting the probe to a manometer or U-tube and then determining the water displacement as the immittance device air pressure dial is rotated. If the SI unit of deca pascals ( $\mathrm{daPa})$ is used, an appropriate measuring device must also be used. The air pressure should not differ from that stated on the device (e.g., $200 \mathrm{daPa}$ ) by more than $\pm 10 \mathrm{daPa}$ or $\pm 15 \%$ of the reading, whichever is greater. The standard states that the air pressure should be measured in cavities with volumes of 0.5 to $2 \mathrm{~cm}^{3}$.

Finally, one should check the reflex activating system. In checking the activation of a contralateral or ipsilateral reflex, normally an insert receiver will be used, which may be measured on a standard HA-1 coupler. The frequency of the activator may be measured electronically directly from the acoustic immittance device. In this case, one uses a frequency counter. Frequency, harmonic distortion, and 
intensity should have tolerances that are similar to those required for an audiometer (ANSI S3.6-2010). That is, frequency should be $\pm 3 \%$ of the stated value, harmonic distortion should be less than $3 \%$ at specified frequencies for earphones and $5 \%$ or less for the probe tube transducer or insert receiver. Noise bands should also be checked if they are to be an activating stimulus. Broadband noises should be uniform within $\pm 5 \mathrm{~dB}$ for the range between 250 and $6,000 \mathrm{~Hz}$ for supra-aural earphones. This can be checked by sending the output through the transducer connected to a coupler, a microphone, and thence to a graphic level recorder or spectrum analyzer. The sound pressure level of tonal activators should be within $\pm 3 \mathrm{~dB}$ of the stated value for frequencies from 250 to $4,000 \mathrm{~Hz}$ and within $\pm 5 \mathrm{~dB}$ for frequencies of 6,000 and $8,000 \mathrm{~Hz}$ and for noise. The rise and fall time should be the same as that described for audiometers and may be measured in the same way. One should have daily listening checks as well as periodic tests of one or two persons with known acoustic immittance to check immittance, tympanogram, and acoustic reflex thresholds to catch any gross problems.

In summary, acoustic immittance devices should be checked as carefully as one's pure tone audiometer. Failure to do so may lead to variability in measurement that may invalidate the immittance measurement.

\section{Sound Source Calibration: Background}

The basic idea behind immittance calibrations is that the ratio of sound pressure and volume velocity produced by a sound source is related to the acoustic "load" impedance that is coupled to the source. With a calibrated sound source, a measurement of sound pressure in an acoustic load can be converted to an estimate of the acoustic immittance of the load. The relationship between source volume velocity and sound pressure is very clear in the cases of ideal sources. For example, an ideal volume velocity source will generate constant volume velocity no matter what load is coupled to the source, so that the ratio of the measured sound pressure produced by the source in an acoustic load and the constant volume velocity define the acoustic impedance of the load.

A reasonable approximation of an ideal volume velocity source is the cam-driven actuator of a pistonphone sound calibrator (7.51: ANSI S1.1-2013). The piston phone creates a near constant volume velocity at a set frequency, which when coupled to a precise rigidly enclosed air volume will produce a precise sound pressure related to the value of the volume displacement and the acoustic impedance associated with the enclosed air volume. However, like all "real" sound sources a pistonphone only approximates the ideal, in that there are circumstances defined by the mechanics and electrics of the piston phone where the volume velocity it generates can vary depending on the acoustic load. For example, in a load such as a waterfilled cavity with very high impedance, the mechanics and electrics of the cam drive may not produce the force necessary to move the piston back and forth at its expected value.

\section{Calibration of the Impedance- Measuring Equipment}

Different impedance-measuring devices require different calibration techniques. Furthermore, these devices are usually calibrated within the factory, and most user-based calibration procedures are really tests of the calibration accuracy. For example, the user will couple a tympanometer sound probe to a $1-\mathrm{cm}^{3}$ acoustic volume and test to see if the measured immittance has a value consistent with that volume; a failure of this test will lead to an investigation of the electrical connections, or inspection of the sound delivery and microphone plumbing for wax or other debris and may lead to a call to the manufacturer for service. The manufacturedescribed calibration test procedure for wideband immittance or reflectance measurement systems is more elaborate and requires measurements in multiple loads, but a failed test usually produces the same result: checks for electrical and acoustic conductivity, and a call to the manufacturer for maintenance and recalibration. For those readers wishing more information on this issue, a detailed description of the calibration procedures is included in an Appendix. 


\section{Determination of Measurement Accuracy}

Paragraph 5.1.5 of IEC 60645-5 states that acoustic immittance measurement systems should be accurate within $\pm 5 \%$, or $\pm 0.1 \mathrm{~cm}^{3}$ or \pm $10^{-9} \mathrm{~m}^{3} / \mathrm{Pa}-\mathrm{s}$, whichever is greater. ${ }^{*}$ Tests of current home-built or commercially available broadband immittance devices generally meet these standards over the frequency range recommended for these devices by each of the manufacturers. Published comparisons of measurements of immittance compared with the theoretical immittance of several kinds of standard loads are illustrated in Rosowski et al, ${ }^{40}$ Allen, ${ }^{41}$ Keefe et al, ${ }^{7}$ Voss and Allen, ${ }^{42}$ and Lynch et al. ${ }^{43}$

\section{SUMMARY}

There is growing emphasis on the use of wideband immittance measurements to aid in the diagnosis and screening of conductive hearing loss (see, e.g., the recent supplement published by Ear and Hearing $\left[\right.$ Feeney $\left.{ }^{44}\right]$ that resulted from extensive discussions of a group of experts in this field). The consensus of this group is that although tympanometric measurements made with one or two pure tones contribute to the detection and diagnosis of middle ear pathology, measurements of immittance and reflectance made over a broader frequency range contribute significant additional information in these areas. Present standards on the measurement of acoustic immittance concentrate on single tone tympanometry and the use of single-tone immittance for testing middle ear muscle reflex. Little is said regarding the calibration of wideband devices, though both the U.S. and International standards require an instruction manual that describes the manufacturer's recommended field calibration procedure. Whether the more elaborate calibration procedures required for wideband immittance and reflectance measurements should be subjected to additional standards is a point for further discussion. A discussion that

\footnotetext{
This is compatible with the spirit of ANSI S3.39-1987 (ANSI 1987), but not its stated limits. There is an inconsistency in section 7.1.5 in the American standard that discusses accuracy of immittance measurement systems. The stated limited \pm 0.1 acoustic mmhos, does not equal $\pm 10^{-9}$ $\mathrm{cm}^{3}-\mathrm{Pa}^{-1}-\mathrm{s}^{-1}$, but does equal $\pm 10^{-9} \mathrm{~m}^{3}-\mathrm{Pa}^{-1}-s^{-1}$ as given in the IEC standard.
}

is already taking place concerns the temporal frequency of such calibrations, where several laboratories endorse the frequent recalibration of wideband immittance measuring devices, and others endorse regular checks to determine if the device is consistent with the factory installed calibration. The manufacturers generally recommend procedures that test the factory calibration of these devices, where failure of such tests lead to simple inspections of the electrical connections and sound tubes in the device, and may result in calls for service. The appendix can add insight into the theory behind the calibration procedure and how one might test the accuracy of immittance and reflectance measuring devices.

\section{ACKNOWLEDGMENTS}

The work involved in understanding impedance and reflectance measurement devices was not performed in a vacuum. We are indebted to our many coworkers over the years, including Bill Peake, Joe Zwislocki, Tom Lynch, and Mike Ravicz and to the pioneering work of Joe Zwislocki, A.S. Feldman, James Jerger, David Lilly, Bob Margolis, and others. Much of that work was also generously supported by The U.S. National Institute of Health via the National Institute of Deafness and other Communicative Disorders and its predecessor The National Institute for Neurological and Communicative Disorders.

\section{REFERENCES}

1. Zwislocki JJ. Some measurements of the impedance at the eardrum. J Acoust Soc Am 1957;29:349-356

2. Zwislocki JJ, Feldman AS. Acoustic impedance of pathological ears. ASHA Monogr 1970;15:1-42

3. Terkildsen K, Thomsen KA. The influence of pressure variations on the impedance of the human ear drum. A method for objective determination of the middle-ear pressure. J Laryngol Otol 1959; 73:409-418

4. Terkildsen K, Nielsen SS. An electroacoustic impedance measuring bridge for clinical use. Arch Otolaryngol 1960;72:339-346

5. Keith RW. Impedance audiometry with neonates. Arch Otolaryngol 1973;97(6):465-467

6. Keith RW. Middle ear function in neonates. Arch Otolaryngol 1975;101(6):376-379

7. Keefe DH, Ling R, Bulen JC. Method to measure acoustic impedance and reflection coefficient. J Acoust Soc Am 1992;91(1):470-485 
8. Djupesland G. Middle ear muscle reflexes elicited by acoustic and nonacoustic stimulation. Acta Otolaryngol Suppl 1964;188(Suppl):188, 287-292

9. Djupesland G. Contraction of the Tympanic Muscles in Man (Thesis). Oslo, Norway: Univeritetsforlaget; 1967

10. Borg E. A quantitative study of the effect of the acoustic stapedius reflex on sound transmission through the middle ear of man. Acta Otolaryngol 1968;66(6):461-472

11. Gelfand SA. The acoustic reflex. In: Katz J, Medwetsky L, Burkard R, Hood LJ, eds. Handbook of Clinical Audiology. Philadelphia PA: Lippincott Williams and Wilkins; 2009:189-221

12. Shahnaz N, Feeney MP, Schairer KS. Wideband acoustic immittance normative data: ethnicity, gender, aging, and instrumentation. Ear Hear 2013;34(Suppl 1):27S-35S

13. Stinson MR, Shaw EAG, Lawton BW. Estimation of acoustical energy reflectance at the eardrum from measurements of pressure distribution in the human ear canal. J Acoust Soc Am 1982;72(3):766-773

14. Keefe DH, Bulen JC, Arehart KH, Burns EM. Ear-canal impedance and reflection coefficient in human infants and adults. J Acoust Soc Am 1993; 94(5):2617-2638

15. Rosowski JJ, Stenfelt S, Lilly D. An overview of wideband immittance measurements techniques and terminology: you say absorbance, I say reflectance. Ear Hear 2013;34(Suppl 1):9S-16S

16. Farmer-Fedor BL, Rabbitt RD. Acoustic intensity, impedance and reflection coefficient in the human ear canal. J Acoust Soc Am 2002;112(2):600-620

17. Rabinowitz WM. Measurement of the acoustic input immittance of the human ear. J Acoust Soc Am 1981;70(4):1025-1035

18. Voss SE, Stenfelt S, Neely ST, Rosowski JJ. Factors that introduce intrasubject variability into ear-canal absorbance measurements. Ear Hear 2013;34 (Suppl 1):60S-64S

19. Lidén G, Peterson JL, Björkman G. Tympanometry. Arch Otolaryngol 1970;92(3):248-257

20. Jerger J. Handbook of Clinical Impedance audiometry. New York, NY: American Electromedics Corporation; 1975

21. Feldman AS, Wilber LA. Acoustic Impedance and Admittance-The Measurement of Middle-Ear Function. Baltimore, MD: Williams \& Wilkins; 1976

22. Shanks JE, Lilly DJ. An evaluation of tympanometric estimates of ear canal volume. J Speech Hear Res 1981;24(4):557-566

23. Keefe DH, Bulen JC, Campbell SL, Burns EM. Pressure transfer function and absorption cross section from the diffuse field to the human infant ear canal. J Acoust Soc Am 1994;95(1):355-371

24. Margolis RH, Paul S, Saly GL, Schachern PA, Keefe DH. Wideband reflectance tympanometry in chinchillas and human. J Acoust Soc Am 2001;110 (3 Pt 1):1453-1464

25. Sanford CA, Feeney MP. Effects of maturation on tympanometric wideband acoustic transfer functions in human infants. J Acoust Soc Am 2008; 124(4):2106-2122

26. Hunter LL, Prieve BA, Kei J, Sanford CA. Pediatric applications of wideband acoustic immittance measures. Ear Hear 2013;34(Suppl 1):36S-42S

27. Prieve BA, Vander Werff KR, Preston JL, Georgantas L. Identification of conductive hearing loss in young infants using tympanometry and wideband reflectance. Ear Hear 2013;34(2): $168-178$

28. Nakajima HH, Pisano DV, Röösli C, et al. Comparison of ear-canal reflectance and umbo velocity in patients with conductive hearing loss: a preliminary study. Ear Hear 2012;33(1):35-43

29. Allen JB, Jeng PS, Levitt H. Evaluation of human middle ear function via an acoustic power assessment. J Rehabil Res Dev 2005;42(4, Suppl 2): 63-78

30. Feeney MP, Keefe DH. Estimating the acoustic reflex threshold from wideband measures of reflectance, admittance, and power. Ear Hear 2001; 22(4):316-332

31. Feeney MP, Keefe DH, Marryott LP. Contralateral acoustic reflex thresholds for tonal activators using wideband energy reflectance and admittance. J Speech Lang Hear Res 2003;46(1):128-136

32. Feeney MP, Keefe DH, Sanford CA. Wideband reflectance measures of the ipsilateral acoustic stapedius reflex threshold. Ear Hear 2004;25(5):421-430

33. Wever EG, Lawrence M. Physiological Acoustics. Princeton, NJ: Princeton University Press; 1954

34. Rabinowitz WM. Acoustic-Reflex Effects on the Input Admittance and Transfer Characteristics of the Human Middle Ear. Ph.D. Thesis.Cambridge MA: Massachusetts Institute of Technology; 1977

35. Peake WT, Rosowski JJ. Acoustic properties of the middle ear. In: Crocker MJ, ed. Encyclopedia of Acoustics. New York, NY: Wiley; 1997:1337-1346

36. Arriaga MA, Luxford WM. Impedance audiometry and iatrogenic hearing loss. Otolaryngol Head Neck Surg 1993;108(1):70-72

37. Hunter LL, Ries DT, Schlauch RS, Levine SC, Ward WD. Safety and clinical performance of acoustic reflex tests. Ear Hear 1999;20(6):506-514

38. Popelka GR, Dubno JR. Comments on the acoustic-reflex response for bone-conducted signals. Acta Otolaryngol 1978;86(1-2):64-70

39. Lilly DJ. Evaluation of the response time of acoustic-immittance instruments. In: Silman S, ed. The Acoustic Reflex. New York, NY: Academic; 1984

40. Rosowski JJ, Peake WT, Lynch TJ III. Acoustic input-admittance of the alligator-lizard ear: nonlinear features. Hear Res 1984;16(3):205-223 
41. Allen JB. Measurements of eardrum acoustic impedance. In: Allen JB, Hall JH, Hubbard A, Neely ST, Tubis A, eds. Peripheral Auditory Mechanisms. New York, NY: Springer-Verlag; 1986: 44-51

42. Voss SE, Allen JB. Measurement of acoustic impedance and reflectance in the human ear canal. J Acoust Soc Am 1994;95(1):372-384

43. Lynch TJ III, Peake WT, Rosowski JJ. Measurements of the acoustic input impedance of cat ears: 10. $\mathrm{Hz}$ to $20 \mathrm{kHz}$. J Acoust Soc Am 1994;96(4): 2184-2209

44. Feeney MP. Editorial: Eriksholm workshop on wideband absorbance measures of the middle ear. Ear Hear 2013;34(Suppl 1):1S-2S

\section{APPENDIX}

\section{The Equivalent Source Description}

There is an analytic technique (Fig. A1) for describing the limitations of real-world sources in terms of the combination of an ideal sound pressure source with output $P_{T}(f)$ and a "series"coupled impedance $Z_{T}(f)$ coupled to a load of variable acoustic impedance $Z_{L}(f)$, where each of these component values can vary with stimulus frequency. The technique depends on an early electrical engineering theorem postulated by a French engineer named Thévenin. Thévenin's theorem relates the sound pressure produced in the load to the sound pressure of the ideal source:

$$
\frac{P_{L}(f)}{P_{T}(f)}=\frac{Z_{L}(f)}{Z_{T}(f)+Z_{L}(f)} . \text { (Eq. A1) }
$$

When the magnitudes of the load and Thévenin impedances differ by more than an order of magnitude, Eq. A1 is consistent with ideal sources: when $\left|Z_{T}(f)\right|$ is much less than | $Z_{L}(f) \mid$, the right-hand side of Eq. A1 approximates 1 and the source acts as a pressure source where $P_{L}(f) \approx P_{T}(f)$ and the measured pressure is independent of $Z_{L}(f){ }^{\dagger}$ When $\left|Z_{T}(f)\right|$ is much greater than $\left|Z_{L}(f)\right|$, the right-hand side of Eq. A1 approximates the ratio of $Z_{L}(f) / Z_{T}(f)$, and $P_{L}(f)$ equals the product of the load impedance and the constant volume velocity produced by the source, where $U_{T}(f) \sim\left(P_{T}(f) / Z_{T}(f)\right)$. Not surprisingly, under many circumstances $\left|Z_{T}(f)\right|$ $\approx\left|Z_{L}(f)\right|$, and the source is neither a sound pressure nor volume velocity source.
45. Egolf DP. Mathematical modeling of a probetube microphone. J Acoust Soc Am 1977; 61:200-205

46. Zuercher JC, Carlson EV, Killion MC. Small acoustic tubes: New approximations including isothermal and viscous effects. J Acoust Soc Am 1988; 83:1653-1660

47. Lynch TJ III. Signal Processing by the Cat Middle Ear: Admittance and Transmission, Measurements and Models. Cambridge MA: Massachusetts Institute of Technology; 1981

48. Beranek LL, Mellow TJ. Acoustics: Sound Fields and Transducers. New York, NY: Academic Press; 2012

\section{Simple Acoustic Loads}

The equivalent source description of real sound sources depends on a series of measurements in known acoustic loads of varied immittance, which then are used to compute the $P_{T}(f)$ and $Z_{T}(f)$ associated with the sound source. In single tone tympanometers, calibration loads are usually closed cavities whose input admittance magnitude is defined by Eq. 1 of the main text. The loads are usually air-filled rigid-walled cylinders with a length 1 to 3 times that of the diameter (10.2 ANSI S3.39 1987). The standard calls for a minimum of three calibration cavities with recommended volumes of $0.5,1$, and $5 \mathrm{~cm}^{3}$; furthermore, the manufactured cavities must have volumes within $3 \%$ of their specified values. According to the standard, the cavities and the sound probe (which couples the sound source and measuring microphone to the ear canal or calibration load) should be designed in a manner that the probe is within a $1 \mathrm{~mm}$ of the cavity opening when the two are coupled together in an airtight manner. The calibration cavities on commercially available tympanometers are generally used only as a check of the equivalent source output. The precise determination of the source parameters for each tympanometer is performed in the factory and hardwired into the electronics and/or digital processor of the device.

\footnotetext{
+ Although an ideal sound pressure source is good for an audiologic earphone, it makes measurements of immittance difficult unless there is a direct measurement of the volume velocity produced by the source.
} 


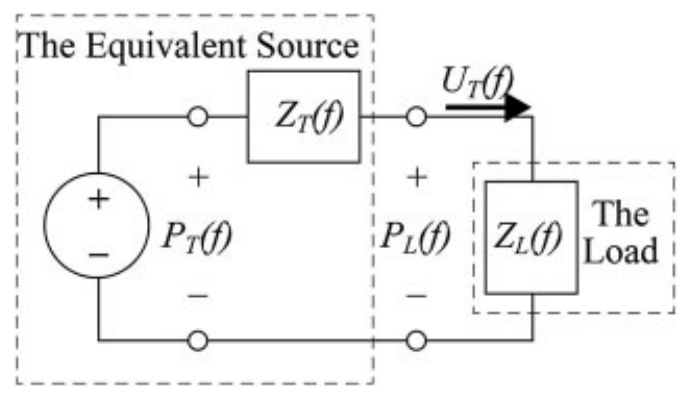

Figure A1 The equivalent source model. The frequency and load dependence of any sound source can be characterized by its equivalent source circuit. The circuit above uses the electroacoustic impedance analogy, ${ }^{48}$ where sound pressure is analogous to an $\mathrm{AC}$ voltage and volume velocity is analogous to electrical current. The source is characterized by the two elements in the large-dashed box on the left (an ideal sound pressure source with output $P_{T}(f)$, and an impedance $\left.Z_{T}(f)\right)$. The source is coupled to a load of acoustic impedance $Z_{L}(f)$. The text describes how the sound pressure measured at the entrance to the acoustic load $P_{L}(f)$ depends on the three other parameters.

Although rigid-walled cavities with an admittance defined by Eq. 1 are useful for testing and calibrating sound sources that measure immittance at low frequencies (e.g., $f<1$ $\mathrm{kHz}$ ), such models are limited at higher frequencies. Eq. 1 is an approximation of a more general description of cavity load admittance that is only valid when the dimensions of the cavity are less than $10 \%$ of the sound wavelength. Ten percent of the wavelength of sound at $1 \mathrm{kHz}$ is $\sim 3.5 \mathrm{~cm}$. With calibration cavities of larger dimensions or at higher frequencies, more complicated descriptions of cavity immittance are required, where precise description depends on the use of simplified geometries. A common geometric simplification is the use of rigidly terminated cylindrical calibration tubes (Fig. A2), where the diameter of the cylindrical opening is generally less than the length of the cylinder (see Rabinowitz ${ }^{17}$; Allen ${ }^{41}$; Keefe et $\mathrm{al}^{7}$ ). In circumstances where sound propagates down the tube as a uniform plane wave, and where we ignore the viscosity and thermal conductivity of air, ${ }^{\ddagger}$ the frequency-dependent input impedance of such an acoustic load $Z_{I}(f)$ depends on the cross-sectional area $S$ and length

\footnotetext{
F The effect of viscosity is to round the peaks and valleys of the impedance magnitude as it varies with frequency, and thermal conductivity has an effect on the impedance at relatively low frequencies. ${ }^{43}$ Descriptions of the impedance of air-filled tubes that include the effects of viscosity and thermal conductivity are available in Egolf ${ }^{45}$ or Zuercher et al. $^{46}$
}

$L$ of the tube, as well as the density $\rho_{0}$ and the propagation velocity of sound $c$ in air:

$$
Z_{j}(f)-i \frac{m_{i 1}}{S}+\left(\frac{2 \pi f}{c} d\right) \quad(1 \mathrm{~kg} \mathrm{~N} \mathrm{j}
$$

Examples of the magnitude and angle of the impedance of several rigidly terminated calibration tubes are illustrated in Fig. A3.

A second type of acoustic load that is simpler in conception and in its impedance, but is somewhat more difficult to realize, is a long tube of uniform cross-section that is terminated by an impedance equal to $Z_{0}$ the acoustic characteristic impedance of the tube. ${ }^{40,43,47}$ In such a tube no sound is reflected from the opposite end, and the input impedance of the tube equals $Z_{0}$. Theoretically, a tube of any length can be made to appear infinite by terminating its far end with an impedance that equals or perfectly matches $Z_{0}$. In reality, any small error in this match will produce reflections that complicate the impedance seen at the input of the tube. The use of a tube of finite but significant length terminated by a matched impedance leads to a reduction in such reflections as the small degree of reflected sound energy is absorbed by viscous interactions with the tube walls along the tubes long length.

The long matched tube impedance has an advantage in that its impedance is nearly invariant with frequency (Fig. A3, tube D), and this impedance is insensitive to small errors in estimating the tube length. On the other hand, the impedances of rigidly terminated tubes 


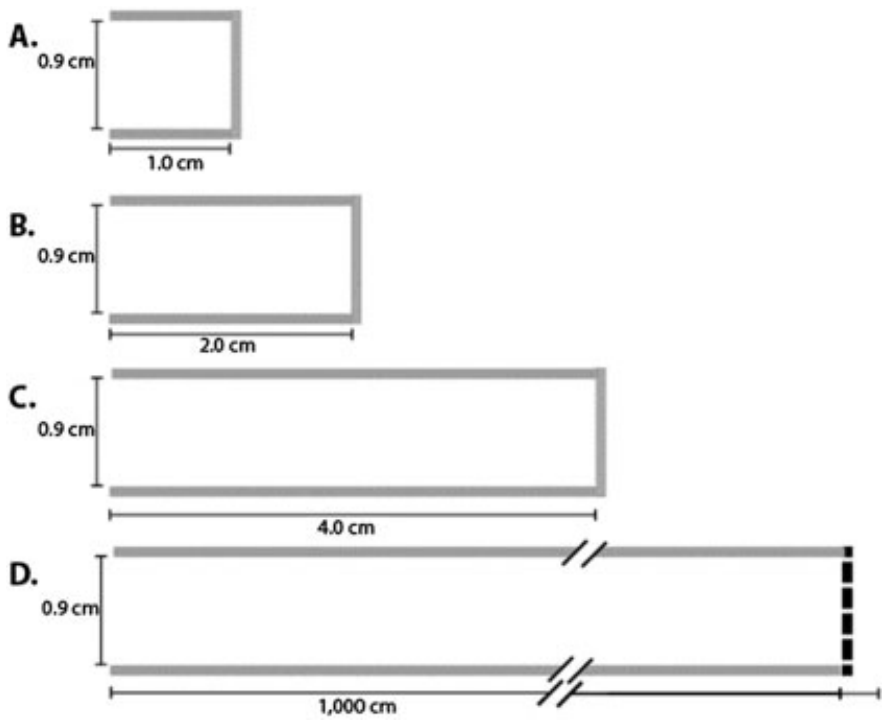

Figure A2 Some simple cylindrical loads. Schematic diagrams of the cross section of four simple acoustic loads of types used in calibrating acoustic impedance sources. (A, B, and C) Three short cylindrical tubes of uniform cross section that are rigidly terminated at their far end. (D) A significantly longer tube that is terminated by an acoustic load (schematized by the dashed black end cap). The acoustic impedance of the terminating load is matched to the characteristic acoustic impedance of the tube (see text description).

(Fig. A2, Tubes A, B, and C) are highly dependent on the tubes' lengths, where the maxima in impedance occur precisely at frequencies where the length of the tubes equals $1 / 4$, $1 \frac{1}{4} \ldots$ of the sound wavelength, and the minima occur at frequencies where the length of the tubes equals $1 / 2,1,1 \frac{1}{2} \ldots$ of the sound wavelength. In rigidly terminated tubes, small errors in estimates of the tube length lead to significant errors in the calculated impedance at frequencies near the minima and maxima.

There is a complication associated with the use of long-matched tubes: they are of relatively low impedance at frequencies below $0.2 \mathrm{kHz}$ (Fig. A3). Because of this low impedance, sound sources with limited low-frequency output may not be able to produced measurable sound at frequencies below $0.2 \mathrm{kHz}$. Some authors have compensated for this potential low-frequency failure by using matched long tubes to characterize sound sources at frequencies above $1 \mathrm{kHz}$, and short rigidly terminated tubes to characterize sources at lower frequencies (e.g., Lynch et $\mathrm{al}^{43,47}$; Rosowski et $\mathrm{al}^{40}$ ). Such an approach has the advantage that the source determination in specific frequency ranges is only performed with acoustic loads with well-defined and regular variations in magnitude and phase that are less affected by small errors in the measurements of tube length or cross-section. Another approach is to overspecify the calibration by measurements in three or more rigidly terminated tubes and determine the source parameters that best fit the calibration data (e.g., Rabinowitz ${ }^{17}$; Allen ${ }^{41}$; Keefe et $\mathrm{al}^{7}$; Voss and Allen ${ }^{42}$; Rosowski et $\mathrm{al}^{15}$ ).

\section{Determination of Equivalent Source Characteristics from Well-Defined Acoustic Loads}

The definition of the equivalent source characteristic requires the measurement of sound pressures in well-defined acoustic loads to determine $P_{\mathrm{T}}(f)$ the equivalent source pressure and $Z_{T}(f)$ the equivalent impedance of the source. Rearrangement of Eq. A1, defines one of the unknown parameters $Z_{T}(f)$ in terms of the sound pressure $P_{L}(f)$ measured at the entrance of a load with known impedance $Z_{L}(f)$ and the second unknown $P_{T}(f)$ :

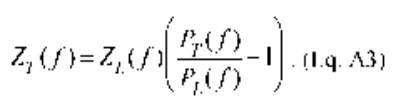

To define the two unknowns, we pair the results from measurements of sound pressure in two 


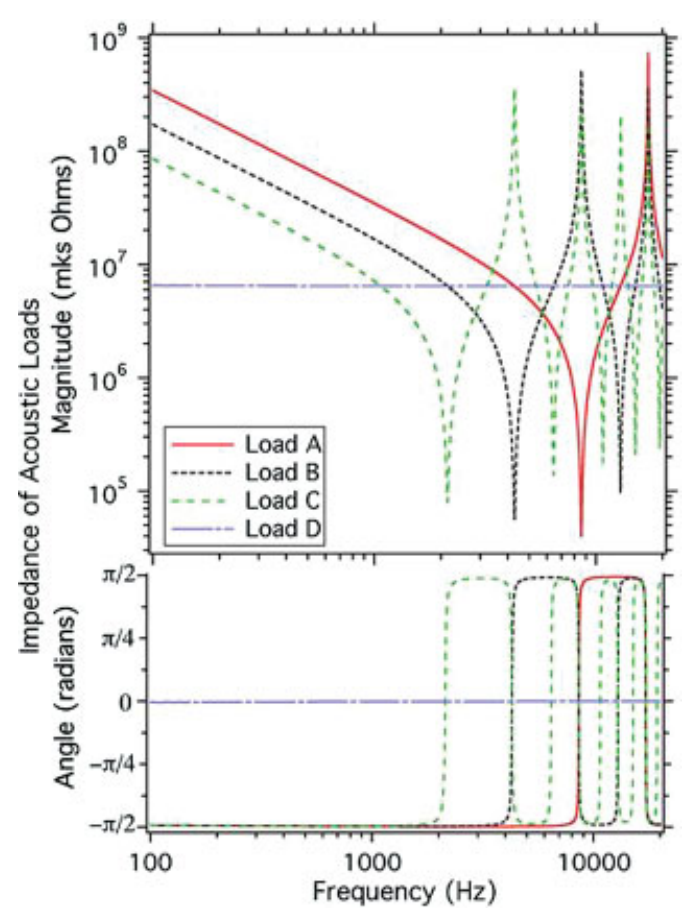

Figure A3 The input impedance of several short, rigidly terminated tubes and an effectively infinite tube. The impedance looking into the open end of the four cylindrical tubes with a radius $a$ of $4.5 \mathrm{~mm}$ in Figure A2. The impedance is calculated for tubes of lengths of 1 (load A), 2 (load B) and 4 (load C) cm that are rigidly terminated at the opposite end (the blue, green dashed, and red dotted lines, respectively). The blue long-short-dashed line is the input impedance of load $D$, a tube $10 \mathrm{~m}$ in length that is terminated with a matched resistance, that is, a resistance equal to the characteristic acoustic impedance of the tube, where $Z_{0}=\rho_{0} c /\left(\pi a^{2}\right)=6.4 \times 10^{6}$ acoustic ohms. The calculations include the contribution of viscosity and thermal conductivity on the impedance. ${ }^{43}$ At frequencies less than $1 \mathrm{kHz}$, the impedances of the rigidly terminated tubes are of larger magnitude; the shortest tube has the largest low frequency impedance. The impedances of each of the rigidly terminated tubes show a series of sharp magnitude peaks and valleys, where the longest tube has the largest number of these extrema. Associated with each peak and valley is a change in phase of $\pm \pi$ radians (180 degrees). The magnitude and angle of the impedance of the longmatched tube (load 4) are nearly constant across the measured frequency range. loads of known impedance $Z_{L 1}(f)$ and $Z_{L 2}(f),{ }^{43}$ such that:

$$
\begin{aligned}
& z_{i}(f)-L_{r_{1}}(f)\left(\frac{P_{r}(f)}{f_{i, 1}(f)}-1\right)-L_{i, 2}(f)\left(\frac{P_{T}(f)}{P_{r_{2}}(f)}-1\right) \\
& \text { which leads to (1:g. A:4] }
\end{aligned}
$$

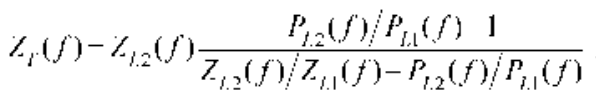

Once $Z_{T}(f)$ is defined, it can be combined with the sound pressure measured in one of the known loads, for example, $P_{L 1}(f)$ and $Z_{L 1}(f)$, and Eq. A1 to compute $P_{T}(f)$ :

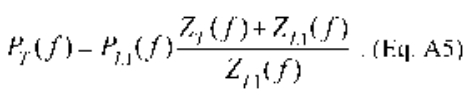

With both $Z_{T}(f)$ and $P_{T}(f)$ defined, we can rearrange Eq. A1 to compute the impedance of an unknown load, for example, in the ear canal, from a measurement of sound pressure in the ear canal $P_{E C}(f)$ :

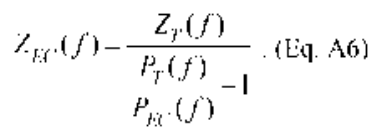

An alternative to Eq. A5 and A6 is to use linear algebra to compute the solution with lowest error for $Z_{T}(f)$ and $P_{T}(f)$ from a set of three or more measurements in different acoustic loads (e.g., Allen $1986^{41}$; Keefe et $\mathrm{al}^{7}$; Rosowski et $\left.\mathrm{al}^{15}\right)$. This technique has the advantage that the effect of small errors in the estimate of the length of rigidly terminated tubes are reduced by the least-squares fitting procedure. 